\title{
Channel Allocation in Multi-user MIMO Wireless Communications Systems
}

\author{
Quentin H. Spencer \\ Distribution Control Systems, Inc. \\ 945 Hornet Dr., Hazelwood, Missouri, 63042, USA \\ qspencer@ieee.org
}

\author{
A. Lee Swindlehurst \\ Dept. of Electrical \& Computer Engineering \\ Brigham Young University, Provo, Utah, 84602, USA \\ swindle@ee.byu .edu
}

\begin{abstract}
The use of multi-user multiple input multiple output (MIMO) processing algorithms in wireless communication systems requires new channel allocation algorithms that can intelligently assign users to channels that can best take advantage of the spatial processing available at both transmitter and receiver. The availability of spatial processing at the receiver adds yet another variable to the classic channel allocation problem, making it very difficult to find the optimal solution for a particular set of users at a reasonable computational cost. We propose a twostep heuristic solution. The first step is the computation of a metric that quantifies the spatial compatibility of two users. The second step is to group the users into shared channels based on optimizing the sum of the compatibility metrics over all groups. We also propose a modified version of the algorithm in which the sub-channels (from different multipath components) of a single user are not required to share the same time-domain channel. In simulations, these algorithms come reasonably close to the optimal solution at a moderate computational cost.
\end{abstract}

\section{INTRODUCTION}

Channel allocation for multi-user mobile wireless networks is a well-studied problem. However, emerging technologies for third and fourth generation networks will add new challenges to the problem, requiring allocation methods that take them into account. In this paper, we look specifically at allocation methods that take into account spatial processing at both base station and mobiles. There has been some research on channel allocation algorithms that take into account the use of adaptive arrays at the base station, but these have assumed that no array processing is used at the mobile devices. The use of array processing at both the transmitter and receiver, also known as multiple input, multiple output (MIMO) processing, has been shown to have significant potential for increases in capacity [1,2], given sufficient multipath diversity. More recently, several algorithms have been proposed for multiuser MIMO processing, where a base station transmits to a group of mobiles who may each have an arbitrary number of antennas. In this paper we propose a channel allocation scheme for such multi-user MIMO channels. We begin by considering the general allocation problem, methods of multiuser multiplexing in MIMO channels, and the problem of combining these two.

\section{A. The Channel Allocation Problem}

Traditionally, the channel allocation problem arises in the context of cellular systems, where the available bandwidth is divided into some set of channels, whether they exist in time domain (TDMA), frequency domain (FDMA), or code domain (CDMA), or some combination of these. The pool of available channels must be allocated to meet the following goals:

- Avoid interference between cells. For example, using the same channel in two adjacent cells can result in a user receiving in-band interference from the other cell.

- Minimize "call blocking", where a user requests a channel and there are none available.

- Maximize total network capacity.

The many algorithms that have been proposed for channel allocation can generally be classified into two categories. The Fixed Channel Allocation (FCA) approach permanently assigns a set of channels to each cell. When all channels are in use, new calls are blocked. Dynamic Channel Allocation (DCA) keeps all channels in a central pool and allows them to be allocated to particular cells as needed. This allows greater flexibility in dealing with fluctuations in the concentrations of users. Generally, FCA algorithms have been shown to be less efficient in the total traffic they can handle, but tend to have a lower blocking probability under heavy loads. Since each of these approaches is more advantageous in certain situations, there are many hybrid schemes which try to combine the desirable attributes of both approaches. A survey of the various FCA, DCA, and hybrid schemes is found in [3].

The use of antenna arrays at base stations give the base the capability of directional transmission and reception, which adds additional complexity to the allocation problem. In the literature, one approach to this problem has been to view array processing only as a means of reducing inter-cell interference [4-6]. Alternatively, intelligent signal processing can be used to achieve Space Division Multiple Access (SDMA), or sharing of a single channel among multiple users by separating them spatially, thus improving total network capacity [7-9]. In this paper we consider the problem of channel allocation for multi-user MIMO channels, where the base-station array uses SDMA algorithms to communicate with a group of users who also have arrays.

\section{B. The Multi-User MIMO Multiplexing Problem}

There have been many recent proposals for SDMA methods in multi-user MIMO communications systems where a base 
station and multiple mobile stations all have arrays of antennas. The theoretical capacity of these channels has been shown to be achievable using novel coding schemes referred to as "writing on dirty paper" [10], but simpler approaches based on beamforming have also been proposed [11,12]. Assuming the transmitter knows the users' channels, the problem is to design appropriate beamforming vectors that avoid interfering with each other, based on different design criteria. These methods will not be able to achieve the same level of performance as dirty-paper coding, but they can come very close in some situations, and have the advantage that they do not depend on what type of coding is used, and can therefore be used with existing communication protocols.

Most of the beamforming algorithms are based on the assumption that the users' channels are independent. In practice, it is highly unlikely that two users' channels will be completely dependent, but even a moderate degree of correlation will at best reduce the system capacity and at worst make the multiplexing problem numerically ill-conditioned.

\section{Problem Definition}

We consider the problem of spatial channel allocation for a single base station when the number of users is greater than the number that can be supported simultaneously on a single channel. To simplify, we assume a quasi-stationary, flatfading channel. Let $n_{T}$ represent the number of transmitters, and $K$ be the number of users. Each user has an arbitrary number of antennas, denoted by $n_{R j}$ for user $j$. We assume a flat fading channel, which for user $j$ is represented by the $n_{R j} \times n_{T}$ matrix $\mathbf{H}_{j}$. Each time-domain channel can be shared by up to $n_{T}$ users, so when $K>n_{T}$, there are more users than can be supported by SDMA alone. Thus, we assume that SMDA is used in conjunction with other multiple access methods (i.e. TDMA, FDMA, CDMA) to increase system capacity. We assume for simplicity that the other multiple access methods do not cause inter-user interference for users that are not sharing a channel. This includes TDMA, and orthogonal FDMA and CDMA methods. Since all of these directly or indirectly separate users by temporal processing, we will use the term "time-domain channels" to refer to the different time, frequency, or code slots available to the base station transmitter.

Given these conditions, the problem we wish to solve is to minimize total transmitted power by choosing which users should share time-domain channels. In the following section, we propose a solution, referred to as the "Compatibility Optimization" algorithm (COA). We then discuss in Section III the application of the COA in situations where multiple sub-channels are being transmitted to a particular user, and we provide simulation results in Section IV.

\section{Compatibility Optimization Algorithm}

Let $K$ be the total number of users in the system, and $G$ the number of groups, which will correspond to the number of time or frequency slots available for multiplexing. The groups are represented as $\mathcal{G}_{1}, \mathcal{G}_{2} \ldots \mathcal{G}_{G}$. The number of users in $\mathcal{G}_{j}$ is $q_{j}$. In order to reduce the complexity of the problem, we impose the restriction that $q_{\min } \leq q_{j} \leq q_{\max }$, where the bounds are defined as:

$$
\begin{gathered}
q_{\max }=\left\lceil\frac{K}{G}\right\rceil \\
q_{\min }=\left\lfloor\frac{K}{G}\right\rfloor .
\end{gathered}
$$

When $K / G$ is an integer, $q_{\max }=q_{\min }$; otherwise $q_{\min }=q_{\max }-$ 1 . While it is theoretically possible for the globally optimal allocation to have minimum and maximum group sizes that are outside these bounds, the probability of this is likely quite low for most cases, and the size restriction allows us to manage the complexity of the problem.

Under these constraints, there will be

$$
G_{\max }=G\left(1-q_{\max }\right)+K
$$

groups containing $q_{\max }$ users, and

$$
G_{\min }=G-G_{\max }
$$

groups containing $q_{\min }$ users. Given these parameters, there exists a total of

$$
\frac{K !}{G_{\max } ! G_{\min } ! \prod_{i=1}^{G} q_{i} !}
$$

possible unique groupings. For example, if $K=4$, and $G=2$, then $q_{1}=q_{2}=2$, and there are a total of 3 unique permutations.

Let $P_{\mathcal{G}_{j}}$ be the required transmitted power for the users in $\mathcal{G}_{j}$. The globally optimal grouping is the one that minimizes

$$
\sum_{j=1}^{G} P_{\mathcal{G}_{j}} .
$$

The problem is that $P_{\mathcal{G}_{j}}$ is a function of both the chosen beamforming strategy and the channels of all users in $\mathcal{G}_{j}$. An individual user's contribution to the required power for any group is a function of its channel's interaction with other users' channels. As a result, the only way to minimize the total transmitted power is to compute $P_{\mathcal{G}_{j}}$ for all possible groupings. For simple cases such as the example above which has 3 unique groupings, it may be realistic to do this, but for any larger number of users, the cost quickly grows too large.

As an alternative, we propose a two-step process for assigning users to groups. The first step is to compute some metric $\xi_{i, j}$ for each pair of users which characterizes the cost of putting users $i$ and $j$ together in the same group. For $K$ users, $K(K-1) / 2$ metric calculations are required. After computing $\xi_{i, j}$ for all possible combinations, a Compatibility Optimization Algorithm (COA) is used to find the grouping is that optimizes

$$
\sum_{g=1}^{G}\left(\sum_{i, j \in \mathcal{G}_{g}} \xi_{i, j}\right) .
$$

The idea of a "compatibility metric" has been proposed previously for systems where all users have one antenna. One 


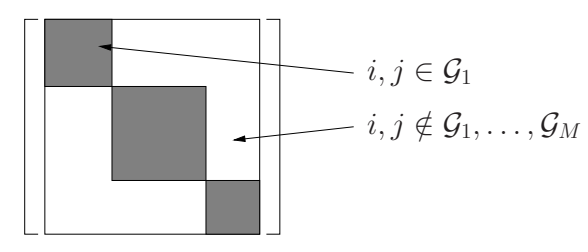

Fig. 1. An illustration of the minimum-sum grouping algorithm.

proposal is a normalized inner product of two users' channels [13]. This is attractive because it requires minimal computation, but it does not simplify to a single constant when the users have multiple antennas. In [7], where the compatibility metric is referred to as a "spatial compatibility check", several different metrics are proposed. The best performing of these is a normalized sum of the interference and noise transmitted to a user. Since computing this as a single scalar also presents problems for multiple-antenna users, a alternative would be to use another metric the authors proposed: the minimum angle between the subspaces spanned by $\mathbf{H}_{i}$ and $\mathbf{H}_{j}$. However, this did not perform as well in empirical tests as the alternative we propose here: a scaled Frobenius norm.

$$
\xi_{i, j}=\frac{\left\|\mathbf{H}_{i} \mathbf{H}_{j}^{*}\right\|_{F}^{2}}{n_{R i} n_{R j}} .
$$

This compatibility metric is effectively an estimate of the total correlation between the two users' channels. The number is scaled by the channel dimensions so that the metric is not unfairly biased against putting users together that have large numbers of receivers, when in reality the additional receivers are an asset that increases SINR when using joint beamforming algorithms.

When the pairwise compatibility metrics have been computed for all cases, it is necessary to find the grouping that minimizes the sum in (5). The problem can be visualized as a matrix $\mathbf{G}$ whose elements $[\mathbf{G}]_{i, j}=\xi_{i, j}$, where $\xi_{i, i}=0$. The matrix $\mathbf{G}$ is illustrated in Figure 1. The sum of the shaded areas is equivalent to twice the sum of (5).

The problem can be characterized in mathematical terms by defining a set of matrices $\mathbf{F}_{1} \ldots \mathbf{F}_{G}$, where $\mathbf{F}_{j}$ is a square matrix of dimension $q_{j}$ (the size of group $G_{j}$ ), consisting of all ones. Define the block-diagonal matrix:

$$
\mathbf{F}=\left[\begin{array}{cccc}
\mathbf{F}_{1} & & & \mathbf{0} \\
& \mathbf{F}_{2} & & \\
& & \ddots & \\
\mathbf{0} & & & \mathbf{F}_{G}
\end{array}\right] .
$$

Let $\mathbf{G}_{0}$ be the matrix of compatibility metrics in arbitrary order. Then the optimal grouping can be characterized as

$$
\begin{aligned}
& \mathbf{P}_{\mathrm{opt}}=\arg \min _{\mathbf{P} \in \mathbf{P}^{(K)}} \mathbf{1}^{T} \mathbf{P}(\mathbf{G} \odot \mathbf{F}) \mathbf{P}^{T} \mathbf{1}, \\
& \mathbf{G}_{\mathrm{opt}}=\mathbf{P}_{\mathrm{opt}} \mathbf{G}_{0} \mathbf{P}_{\mathrm{opt}}^{T}
\end{aligned}
$$

where $\mathbf{P}^{(K)}$ represents the set of all permutation matrices of dimension $K \times K$ and $\mathbf{1}$ represents a vector consisting of all ones, also of dimension $K$. An algorithm for finding $\mathbf{P}_{\text {opt }}$ is outlined below.

\section{Compatibility Optimization Algorithm}

1) Initialization

(i) Given $G$ and $K$, compute $q_{\max }, q_{\min }, G_{\max }$, and $G_{\min }$.

(ii) Generate $\mathbf{F}$ for the channel dimensions

(iii) Determine an arbitrary $\mathbf{G}_{0}$ and compute $s_{0}=$ $\mathbf{1}^{T}\left(\mathbf{G}_{0} \odot \mathbf{F}\right) \mathbf{1}$.

(iv) Generate the set of $K(K-1) / 2$ pairs of integers taken from the set $\{1 \ldots K\}$.

(v) Let $\mathbf{P}_{\text {opt }}=\mathbf{I}_{K}$.

2) For each pair $i, j \in\{1 \ldots K\}$

(i) Let $\mathbf{P}_{i, j}$ be the permutation matrix generated by switching columns $i$ and $j$ of $\mathbf{I}_{K}$.

(ii) If $\mathbf{1}^{T} \mathbf{P}_{i, j} \mathbf{P}_{\text {opt }}(\mathbf{G} \odot \mathbf{F}) \mathbf{P}_{\text {opt }}^{T} \mathbf{P}_{i, j}^{T} \mathbf{1}<0$, let $\mathbf{P}_{\text {opt }}=$ $\mathbf{P}_{i, j} \mathbf{P}_{\text {opt }}$

The result of this procedure is that the permutation matrix $\mathbf{P}_{\text {opt }}$ combined with the initial ordering of the users in $\mathbf{G}_{0}$ reveals the grouping that minimizes the sum of the compatibility metrics. If some metric is used that should be maximized rather than minimized, the only modification required is to change the direction of inequality in step 2(ii).

In computing the inequality in step 2(ii), it is important to note that the only difference between $\mathbf{P}_{i, j} \mathbf{P}_{\text {opt }}(\mathbf{G} \odot \mathbf{F}) \mathbf{P}_{\text {opt }}^{T} \mathbf{P}_{i, j}^{T}$ and $\mathbf{P}_{\text {opt }}(\mathbf{G} \odot \mathbf{F}) \mathbf{P}_{\text {opt }}^{T}$ is the switching of two rows and columns. As a result, an efficient implementation of the inequality can be devised that only computes the sums of the rows and columns in question. The Compatibility Optimization Algorithm requires all $K(K-1) / 2$ steps to be completed to find the global minimum. As noted before, there are $K(K-$ 1) $/ 2$ compatibility metrics to be computed to generate the matrix $\mathbf{G}_{0}$. So, both of the two steps have $\mathcal{O}\left(K^{2}\right)$ complexity.

After a grouping has been selected, it is still necessary to implement a spatial multiplexing algorithm to generate transmit vectors for each group. The Compatibility Optimization Algorithm is independent of which algorithm is chosen, but the compatibility metric should is not necessarily. The correlation metric has been suggested because it worked best with the cases that were tested in the simulations section that follows, but it is possible that for a particular choice of multiplexing algorithm, an alternative metric may yield better performance.

\section{Sub-Channel Allocation}

Up to this point, our discussion of channel allocation has focused on the problem of choosing the best grouping of users without regard to the number of spatial "sub-channels" (SSC) being used by each user. One of the fundamental trade-offs in the design of any MIMO system is whether to maximize transmission rate, which is achieved by parallel data transmission schemes, or to maximize diversity, using spacetime codes [14]. For channels where the transmitter knows the transfer function in advance, one approach to parallel data transmission is to compute the singular value decomposition of the channel matrix, using the right and left singular vectors 


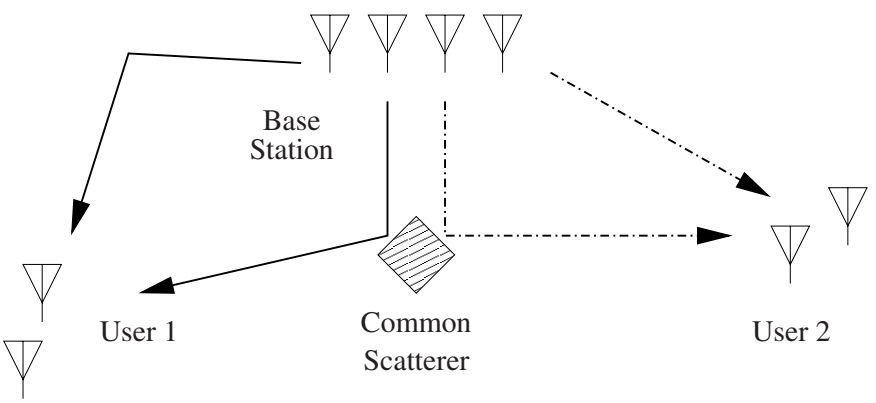

Fig. 2. An illustration of a base station transmitting over multiple subchannels to two users.

as transmit and receive beamformers to create independent, orthogonal SSC [2]. We now consider the question of optimal allocation in a multi-user MIMO channel where multiple SSC are used.

Some of the recent proposed downlink multiplexing algorithms for MU-MIMO systems have taken a simplified approach that allows for the transmission of only one data stream to each user, while there have been some algorithms proposed that allow multiple data streams. The problem of optimal grouping when using a multi-stream transmission scheme raises interesting questions about channel allocation. This is not really an issue when the system is fully loaded $(K=G)$, since there is no excess capacity in the system to allow for the transmission of multiple SSC to individual users. On the other hand, when $K \ll n_{T} G$, the demands on system resources can be reduced by allocating free SSC to users whose channel characteristics will support multiple SSC.

The simplest way to handle the allocation of multiple SSC is to group the users using the algorithm outlined in the last section, and then determine within each group how many SSC are allocated to each user. However, suppose users $i$ and $j$ have been assigned to the same group, and both users have sufficient channel rank to support transmission of data over two SSC. While the two users may have sufficient linear independence to be grouped together when only transmitting one data substream to each user, it my be possible that only some of the SSC of one user are "spatially compatible" with the SSC of the other user. One example of this is illustrated in Figure 2 , where users 1 and 2 share a common scatterer in their multipath channels. In this situation, it is possible that better system resource usage could be achieved by allowing the two SSC of users 1 and 2 to be allocated to different groups.

To accomplish this, we propose an independent sub-channel approach to allocation, where the SSC of each user are treated as completely independent channels in the allocation algorithm. Let $m_{j}$ be the number of sub-channels allocated to user $j$, and let $m=\sum m_{j}$. So, for a system of $K$ users, the $m$ total sub-channels are instead treated as independent channels in the allocation algorithm, increasing the complexity of the allocation algorithm to $\mathcal{O}\left(\mathrm{m}^{2}\right)$. This requires a different compatibility metric, since the correlation metric reflects only

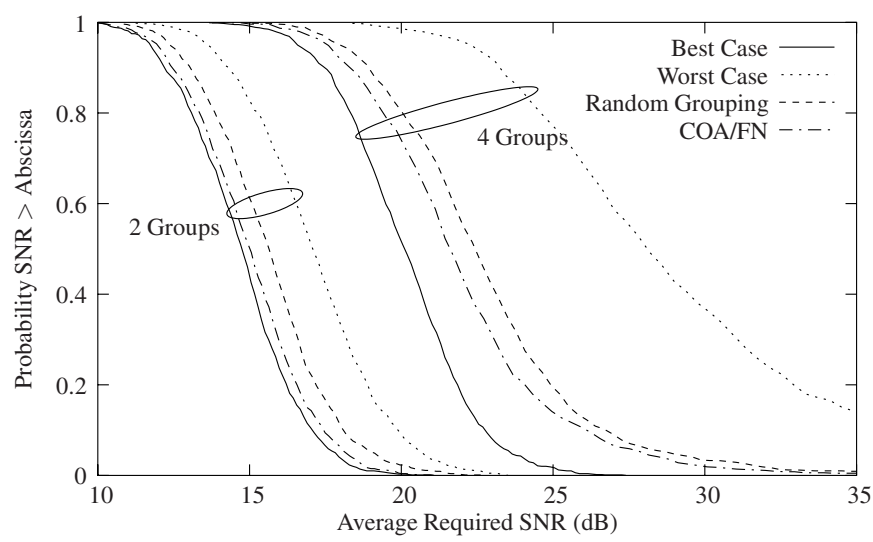

Fig. 3. A comparison of different grouping algorithms for eight users allocated with a set of either two or four available channels.

the compatibility between the entire $\mathbf{H}_{i}$ and $\mathbf{H}_{j}$, rather than their sub-channels. We propose computing the SVD of $\mathbf{H}_{j}$. We find the water-filling solution that minimizes transmitted power given the singular values of $\mathbf{H}_{j}$ and the desired transmission rate, and choose $m_{j}$ as the number of channels with non-zero power. Then, we use the left singular vectors of $\mathbf{H}_{j}$ as a means of estimating spatial compatibility between sub-channels of users. If $\mathbf{w}_{i, k}$ is the $k^{\text {th }}$ left singular vector of $\mathbf{H}_{i}$, then let

$$
\mathbf{w}_{i, k}^{*} \mathbf{H}_{i} \mathbf{H}_{j}^{*} \mathbf{w}_{j, l}
$$

be the spatial compatibility of sub-channel $k$ of user $i$ and sub-channel $l$ of user $j$. Note that the orthogonality of the left singular vectors of a particular $\mathbf{H}_{j}$ results in a compatibility metric of zero when $i=j$. This will bias the COA toward putting the sub-channels of the same user in the same group. However, in simulations it was frequently observed that subchannels of the same user were often allocated to different groups in spite of this, due to one of the sub-channels having sufficiently bad compatibility with other potential members of the group.

\section{Simulation Results}

Figure 3 compares the performance of different channel allocation schemes. In this case, there are a total of four antennas at the base station and eight users with the number of antennas at each user chosen randomly from one to three. A single-beam algorithm is used to transmit to each user, and the transmission rate for each user is a randomly chosen integer in the interval $[1,4]$ bits per user. The number of bits per channel was mapped to a SINR requirement $\left(\gamma_{b}\right)$ using the upper bound on the symbol error rate for uncoded QAM constellations from [15] (equation 5-2-80):

$$
P_{M} \leq 2 \operatorname{erfc}\left(\sqrt{\frac{3 k}{2(M-1)} \gamma_{b}}\right),
$$

where $k$ is the number of bits, $M=2^{k}$, and $\gamma_{b}$ is the average SNR per bit. The required bit error rate was fixed at $10^{-5}$. We consider two different channel configurations, where the eight users are allocated among either two or 


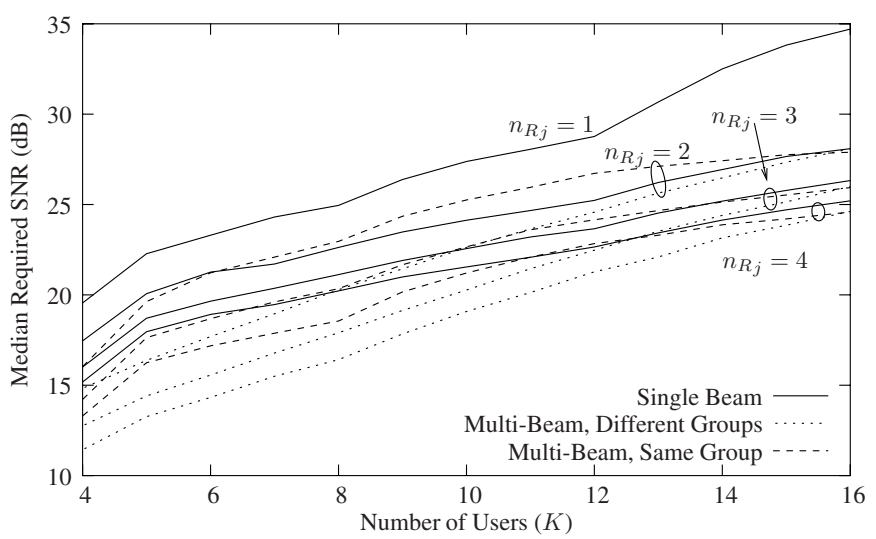

Fig. 4. A comparison of sub-channel grouping algorithms.

four available channels. For two channels, there are a total of 35 unique groupings, and for four channels there are a total of 105 unique groupings. The required power to satisfy the requested rate was calculated for each of the unique groupings in order to determine the best and worst cases. The Compatibility Optimization Algorithm is used in conjunction with the Frobenius norm metric, and is labeled "COA/FN".

The results in Figure 3 reveal that the Compatibility Optimization Algorithm has a cost between the best possible grouping and randomly chosen grouping for both group sizes. It should also be noted that the randomly chosen grouping has surprisingly good performance when compared to the worst case.

Figure 4 compares three different approaches to sub-channel allocation as a function of system loading. In this case the total number of groups is fixed at four, and the number of transmitters at the base station is also four, resulting in a maximum supportable user load of 16 . The number of users was varied from 4, which means only one user per group for the single-beam approach, up to 16 , representing a fully loaded system. In addition to the single-beam algorithm, the multibeam algorithm was tested for two different cases: one using the same grouping as the single-beam algorithm, but allowing additional sub-channels in cases where it was supported, and the second treating all sub-channels as independent channels and performing an independent channel allocation using the metric suggested in the last section. We include results in the plot for values of $n_{R j}$ from one to four antennas. When $n_{R j}=1$ for all users, only one sub-channel per user is available, so the results are the same in all cases.

The results show that the best algorithm is a function of how loaded the system is. Surprisingly, there is minimal advantage gained from allowing multiple sub-channels and requiring them to be in the same group. On the other hand, while all of the results are very close as the system becomes fully loaded, the independent sub-channel approach becomes increasingly superior as the load decreases, by as much as $4 \mathrm{~dB}$. This illustrates the fact that the optimal solution to the channel allocation problem will not necessarily be one that requires all sub-channels for a particular user to be used simultaneously.

\section{CONCLUSION}

We have illustrated the problem of channel allocation in multi-user MIMO channels when there are more users than can be supported using only SDMA methods, and have proposed an algorithm for assigning users to groups. Simulation results have demonstrated that the Compatibility Optimization Algorithm presented here will choose groupings that are somewhat better than random groupings, but not necessarily the global optimum. The optimal solution to this problem can only be found by global search, which is not a realistic option for any significant number of users. The COA is therefore a reasonable alternative.

We have also studied the problem of channel allocation when multiplexing algorithms are used that allow multiple subchannels for individual users. The simulation results show that the best possible solution is often achieved when a user's subchannels are allowed to be allocated to different groups, being treated as independent channels.

\section{REFERENCES}

[1] E. Telatar, "Capacity of multi-antenna gaussian channels," European Trans. on Telecommunications, vol. 10, no. 6, Nov./Dec. 1999.

[2] G. G. Raleigh and J. M. Cioffi, "Spatio-temporal coding for wireless communication," IEEE Trans. Comm., vol. 46, no. 3, pp. 357-366, Mar. 1998.

[3] I. Katzela and M. Naghshineh, "Channel assignment schemes for cellular mobile telecommunication systems: a comprehensive survey," IEEE Personal Communications, vol. 3, no. 3, pp. 10-31, June 1996.

[4] J. S. Blogh, P. J. Cherriman, and L. Hanzo, "Adaptive antenna array assited dynamic channel allocation techniques," IEEE Journal on Selected Areas in Communications, vol. 19, no. 2, pp. 305-311, Feb. 2001.

[5] L. Chen, H. Murata, S. Yoshida, and S. Hirose, "A dynamic channel assignment algorithm for cellular system with adaptive array antennas," in Proc. IEEE Vehicular Tech. Conf., Spring 1999, vol. 1, pp. 204-208.

[6] A. Alexiou and R.-H. Yan, "Downlink capacity enhancement by employing SDMA in GSM," in Proc. 2000 IEEE Sensor Array and Multichannel Sig. Proc. Workshop, pp. 413-417.

[7] C. Farsakh and J. A. Nossek, "A real time downlink channel allocation scheme for an SDMA mobile radio system," in Proc. 7th IEEE Symp. on Personal, Indoor and Mobile Radio Communications (PIMRC), vol. 3, Oct. 1996, pp. 1216-1220.

[8] S. Harano, Y. Akaiwa, and J. Uchibori, "Performance of dynamic channel assignment methods in cellular systems using beam tilting and adaptive array," in Proc. IEEE Vehicular Technology Conf., Fall 1999, vol. 4, pp. 2092-2095.

[9] L. C. Godara, "Applications of antenna arrays to mobile communications. I. performance improvement, feasibility, and system considerations," Proc. IEEE, vol. 85, no. 7, pp. 1031-1060, July 1997.

[10] W. Yu and J. Cioffi, "Sum capacity of gaussian vector broadcast channels," Nov. 2001, submitted to IEEE Trans. on Inform. Theory.

[11] Q. H. Spencer, A. L. Swindlehurst, and M. Haardt, "Zero-forcing methods for downlink spatial multiplexing in multi-user MIMO channels," IEEE Trans. on Sig. Proc., vol. 52, no. 2, Feb. 2004.

[12] — "Fast power minimization with QoS constraints in multi-user MIMO downlinks," in Proc. IEEE Int. Conf. on Acoustics, Speech, and Signal Processing. April 2003.

[13] T. Ohgane, Y. Ogawa, and K. Itoh, "A study on a channel allocation scheme with an adaptive array in SDMA," in Proc. 1997 IEEE Vehicular Technology Conf., vol. 2, pp. 725-729.

[14] A. Goldsmith, S. A. Jafar, N. Jindal, and S. Vishwanath, "capacity limits of MIMO channels," IEEE Journal on Selected Areas in Communications, vol. 21, no. 5, pp. 684-702, June 2003.

[15] J. G. Proakis, Digital Communications, 3rd ed. McGraw Hill, 1989. 